\title{
ON THE GROWTH OF TORSION IN THE COHOMOLOGY OF ARITHMETIC GROUPS
}

\author{
WERNER MÜLLER AND JONATHAN PFAFF
}

\begin{abstract}
In this paper we consider certain families of arithmetic subgroups of $\mathrm{SO}^{0}(p, q)$ and $\mathrm{SL}_{3}(\mathbb{R})$, respectively. We study the cohomology of such arithmetic groups with coefficients in arithmetically defined modules. We show that for natural sequences of such modules the torsion in the cohomology grows exponentially.
\end{abstract}

\section{INTRODUCTION}

Let $G$ be a semi-simple connected algebraic group over $\mathbb{Q}, K$ a maximal compact subgroup of its group of real points $G(\mathbb{R})$. Let $\widetilde{X}=G(\mathbb{R}) / K$ be the associated Riemannian symmetric space. Let $\mathfrak{g}$ and $\mathfrak{k}$ be the Lie algebras of $G(\mathbb{R})$ and $K$, respectively. Put $\delta(\widetilde{X})=\operatorname{rank}\left(\mathfrak{g}_{\mathbb{C}}\right)-\operatorname{rank}\left(\mathfrak{k}_{\mathbb{C}}\right)$. Sometimes $\delta(\widetilde{X})$ is called the fundamental rank. Let $\Gamma \subset G(\mathbb{Q})$ be an arithmetic subgroup and $X=\Gamma \backslash \widetilde{X}$ the corresponding locally symmetric space. We assume that $G$ is anisotropic over $\mathbb{Q}$, which implies that $\Gamma$ is cocompact in $G(\mathbb{R})$. Let $M$ be an arithmetic $\Gamma$-module, which means that $M$ is a finite rank free $\mathbb{Z}$-module, and there exists an algebraic representation of $G$ on $M \otimes_{\mathbb{Z}} \mathbb{Q}$ such that $\Gamma$ preserves $M$. Then the cohomology $H^{*}(\Gamma, M)$ is a finite rank $\mathbb{Z}$-module. Note that if $\Gamma$ is torsion free, then $H^{*}(\Gamma, M) \cong H^{*}(\Gamma \backslash \widetilde{X}, \mathcal{M})$, where $\mathcal{M}$ is the local system of free $\mathbb{Z}$-modules associated to $M$.

For arithmetic reasons, one expects that if $\delta(\widetilde{X})=0$, there is little torsion in $H^{*}(\Gamma, M)$ and the free part dominates the cohomology. On the other hand, if $\delta(\widetilde{X})=1$, one expects a lot of torsion in the cohomology and the free part to be small. This has been substantiated by Bergeron and Venkatesh [BV], who studied the growth of the torsion if $\Gamma$ varies through a sequence of congrunce subgroups $\Gamma_{n}$ for which the injectivity radius of $\Gamma_{n} \backslash \tilde{X}$ goes to infinity. They showed that if $\delta(\tilde{X})=1$ and $M$ is strongly acylic, the torsion grows exponentially proportional to the volume of $\Gamma_{n} \backslash \tilde{X}$. Furthermore, for compact oriented hyperbolic 3manifolds, in MaMü the growth of the torsion has been studied if $\Gamma$ is fixed but the $\Gamma$-module $M$ grows. More precisely, let $X=\Gamma \backslash \mathbb{H}^{3}$ be a compact, oriented hyperbolic 3 -manifold with $\Gamma \subset \mathrm{SL}(2, \mathbb{C})$. Let $V_{m}$ be the holomorphic irreducible representation of $\mathrm{SL}(2, \mathbb{C})$ of dimension $m+1$. By $\mathrm{BW}$ one has $H^{*}\left(\Gamma, V_{m}\right)=0$. It was proved in MaMü that for each even $k \in \mathbb{N}$ there exists a $\Gamma$-invariant lattice $M_{k} \subset V_{k}$. Then $H^{p}\left(\Gamma, M_{k}\right)$ is a

Date: October 1, 2018.

1991 Mathematics Subject Classification. Primary: 11F75.

Key words and phrases. Arithmetic groups, cohomology. 
finite abelian group for all $p$, and the main result of [MaMü is the following asymptotic formula

$$
\lim _{k \rightarrow \infty} \frac{\log \left|H^{2}\left(\Gamma, M_{2 k}\right)\right|}{k^{2}}=\frac{2}{\pi} \operatorname{vol}(X)
$$

and the estimation

$$
\log \left|H^{p}\left(\Gamma, M_{2 k}\right)\right| \ll k \log k, \quad p=1,3 .
$$

Note that $H^{0}\left(\Gamma, M_{2 k}\right)=0$.

The goal of the present paper is to study the growth of the torsion if $M$ varies, for all compact arithmetic quotients $\Gamma \backslash \tilde{X}$ of irreducible symmetric spaces $\tilde{X}$ with $\delta(\tilde{X})=1$. By the classification of simple Lie groups, the irreducible symmetric spaces with $\delta(\tilde{X})=1$ are $\widetilde{X}=\mathrm{SO}^{0}(p, q) / \mathrm{SO}(p) \times \mathrm{SO}(q)$, for $p, q$ odd, and $\widetilde{X}=\mathrm{SL}(3, \mathbb{R}) / \mathrm{SO}(3)$.

The first family of arithmetic groups that we consider are cocompact arithmetic subgroups of $\mathrm{SO}^{0}(p, q)$ that arise from quadratic forms over totally real number fields. More precisely, let $F$ be a totally real finite Galois extension of $\mathbb{Q}$ of degree $d>1$. We fix an embedding $F \subset \mathbb{R}$. Let $Q: \mathbb{R}^{p+q} \rightarrow \mathbb{R}$ be a non-degenerate quadratic form defined over $F$ of signature $(p, q)$. Assume that all non-trivial Galois conjugates of $Q$ are positive definite. Let $G:=\mathrm{SO}_{Q} \subset \mathrm{GL}_{p+q}$ be the special orthogonal group of $Q$, i.e., the subgroup of all elements of determinant one leaving $Q$ invariant. This is a connected algebraic group over $F$ and its group of real points $G(\mathbb{R})$ is isomorphic to $\operatorname{SO}(p, q)$.

Let $\mathcal{O}_{F}$ be the ring of algebraic integers of $F$, and let $G_{\mathcal{O}_{F}}$ be the group of $\mathcal{O}_{F}$-valued points of $G$. Then $G_{\mathcal{O}_{F}}$ is a discrete cocompact subgroup of $G(\mathbb{R})$. Via the isomorphism $G(\mathbb{R}) \cong \mathrm{SO}(p, q)$, it corresponds to a discrete cocompact subgroup $\Gamma_{0}$ of $\mathrm{SO}(p, q)$ (see section 3). If we pass to an appropriate subgroup of finite index $\Gamma \subset \Gamma_{0}$, we may assume that $\Gamma$ is torsion free and that it is containd in $\operatorname{SO}^{0}(p, q)$.

Since $G$ is only defined over $F$, we need to generalize the notion of an arithmetic $\Gamma$ module. Let $G^{\prime}=\operatorname{Res}_{F / \mathbb{Q}}(G)$ be the algebraic $\mathbb{Q}$-group obtained from $G$ by restriction of scalars. Let $\Delta: G \rightarrow G^{\prime}$ be the diagonal embedding. Consider $\Gamma$ as a subgroup of $G_{\mathcal{O}_{F}}$. Let $\Gamma^{\prime}=\Delta(\Gamma)$. Then $\Gamma^{\prime} \subset G^{\prime}(\mathbb{Q})$ is an arithmetic subgroup. Let $M$ be an arithmetic $\Gamma^{\prime}$-module. Since $\Gamma \cong \Gamma^{\prime}$, it becomes a $\Gamma$-module and we also call it an arithmetic $\Gamma$-module.

To state our main result, we need to introduce some notation. Let $\widetilde{X}^{d}$ be the compact dual symmetric space of $\tilde{X}$. We chose an $\mathrm{SO}^{0}(p, q)$-invariant metric on $\tilde{X}$ and equip $X$ and $\widetilde{X}^{d}$ with the induced metrics. Assume that $p, q$ are odd, $p \geq q, p>1$ and let $n:=(p+q) / 2-1$. We let $\epsilon(q):=0$ for $q=1$ and $\epsilon(q):=1$ for $q>1$ and we put

$$
C_{p, q}=\frac{(-1)^{\frac{p q-1}{2}} 2^{\epsilon(q)} \pi}{\operatorname{vol}\left(\widetilde{X}^{d}\right)}\left(\begin{array}{c}
n \\
\frac{p-1}{2}
\end{array}\right) .
$$

Then our first main result is the following theorem.

Theorem 1.1. Let $F$ be a totally real Galois extension of $\mathbb{Q}$ of degree $d>1$. Let $\Gamma$ be a torsion free cocompact arithmetic subgroup of $\mathrm{SO}^{0}(p, q)$ derived from a quadratic form $Q$ over $F$ as above. Then there exists a sequence of arithmetic $\Gamma$-modules $M_{m}, m \in \mathbb{N}$, with 
the following properties. The rank $\operatorname{rk}_{\mathbb{Z}}\left(M_{m}\right)$ is a polynomial in $m$ and there exists $C>0$, which depends only on $n$, such that

$$
\operatorname{rk}_{\mathbb{Z}}\left(M_{m}\right)=C d m^{d n(n+1) / 2}+O\left(m^{d n(n+1) / 2-1}\right)
$$

as $m \rightarrow \infty$. Furthermore each cohomology group $H^{j}\left(\Gamma, M_{m}\right)$ is finite and

$$
\sum_{j \geq 0}(-1)^{j} \log \left|H^{j}\left(\Gamma, M_{m}\right)\right|=-C_{p, q} \operatorname{vol}(\Gamma \backslash \tilde{X}) m \operatorname{rk}_{\mathbb{Z}}\left(M_{m}\right)+O\left(\operatorname{rk}_{\mathbb{Z}}\left(M_{m}\right)\right)
$$

as $m \rightarrow \infty$.

Let $k=(\operatorname{dim}(\tilde{X})+1) / 2$. Then it follows from Theorem 1.1 that there exists a constant $\tilde{C}_{p, q}>0$, which depends only on $p, q$, such that

$$
\liminf _{m} \sum_{j \equiv k(2)} \frac{\log \left|H^{j}\left(\Gamma, M_{m}\right)\right|}{m^{d n(n+1) / 2+1}} \geq \tilde{C}_{p, q} d \operatorname{vol}(\Gamma \backslash \tilde{X}) .
$$

Thus there is at least one $j$ for which $\left|H^{j}\left(\Gamma, M_{m}\right)\right|$ grows exponentially in $m$. Given (1.1) and (1.2), one is tempted to pose the following conjecture.

Conjecture 1.2. Let $\Gamma$ and $M_{m}, m \in \mathbb{N}$, be as above. Then

$$
\lim _{m \rightarrow \infty} \frac{\log \left|H^{j}\left(\Gamma, M_{m}\right)\right|}{m^{d n(n+1) / 2+1}}= \begin{cases}\tilde{C}_{p, q} d \operatorname{vol}(\Gamma \backslash \tilde{X}), & j=(\operatorname{dim}(\tilde{X})+1) / 2, \\ 0, & \text { else. }\end{cases}
$$

The next case that we consider are arithmetic subgroups of $\mathrm{SL}_{3}(\mathbb{R})$ which arise from 9-dimensional division algebras $D$ over $\mathbb{Q}$. Let $\mathfrak{o}$ be an order in $D$. Then $\mathfrak{o}$ induces an arithmetic subgroup $\Gamma$ of $\mathrm{SL}_{3}(\mathbb{R})$ which is cocompact (see section $\mathbb{1}$ ). After passing to a subgroup of finite index, we may assume that $\Gamma$ is torsion-free.

Let $\mathfrak{t}_{\mathbb{C}}$ be the standard complexified Cartan-subalgebra of the Lie algebra of $\mathrm{SL}_{3}(\mathbb{R})$ equipped with the standard ordering of the roots and let $\omega_{1}, \omega_{2} \in \mathfrak{t}_{\mathbb{C}}^{*}$ be the corresponding fundamental weights, see (4.30). If $\Lambda=\tau_{1} \omega_{1}+\tau_{2} \omega_{2} \in \mathfrak{t}_{\mathbb{C}}^{*}, \tau_{1}, \tau_{2} \in \mathbb{N}$ is a dominant weight and $\tau_{\Lambda}$ is the corresponding irreducible finite-dimensional representation of $\mathrm{SL}_{3}(\mathbb{R})$, we let $\Lambda_{\theta}$ be the highest weight of $\tau_{\Lambda} \circ \theta$. One has $\Lambda_{\theta}=\tau_{2} \omega_{1}+\tau_{1} \omega_{2}$. Moreover, for $m \in \mathbb{N}$ we let $\tau_{\Lambda}(m)$ be the irreducible representation of $\mathrm{SL}(3, \mathbb{R})$ on a complex vector space $V_{\Lambda}(m)$ with highest weight $m \Lambda$. We regard $V_{\Lambda}(m)$ as a real vector-space. Let $\widetilde{X}=\operatorname{SL}(3, \mathbb{R}) / \operatorname{SO}(3)$, let $\widetilde{X}_{d}$ be the compact dual of $\tilde{X}$ and let $X=\Gamma \backslash \widetilde{X}$. We fix a $G$-invariant metric on $\widetilde{X}$ which induces metrics on $X$ and on $\widetilde{X}_{d}$. Then our main result for the $\mathrm{SL}_{3}(\mathbb{R})$-case is the following theorem.

Theorem 1.3. Let $\Gamma$ be an arithmetic subgroup of $\mathrm{SL}_{3}(\mathbb{R})$ which arises from a 9-dimensional division algebra over $\mathbb{Q}$. Let $\Lambda \in \mathfrak{t}_{\mathbb{C}}^{*}$ be a highest weight with $\Lambda_{\theta} \neq \Lambda$. Then for each $m$ there exists a lattice $M_{\Lambda}(m)$ in $V_{\Lambda}(m)$ which is stable under $\Gamma$. Moreover, each cohomology 
group $H^{p}\left(\Gamma, M_{\Lambda}(m)\right)$ is finite and one has

$$
\begin{aligned}
\sum_{p=0}^{5}(-1)^{p} & \log \left|H^{p}\left(\Gamma, M_{\Lambda}(m)\right)\right| \\
& =-\frac{\pi \operatorname{vol}(X)}{\operatorname{vol}\left(\widetilde{X}_{d}\right)} C(\Lambda) m \cdot \mathrm{rk}_{\mathbb{Z}} M_{\Lambda}(m)+O\left(\operatorname{rk}_{\mathbb{Z}} M_{\Lambda}(m)\right),
\end{aligned}
$$

as $m \rightarrow \infty$, where $C(\Lambda)>0$ is a constant which depends only on $\Lambda$. If $\Lambda$ equals one of the fundamental weights $\omega_{f}$ then $C(\Lambda)=4 / 9$.

The rank of $M_{\Lambda}(m)$ can be computed explicitly as follows. Firstly, if $\Lambda$ is equal to one of the fundamental weights $\omega_{1}$ or $\omega_{2}$, then $\Lambda_{\theta} \neq \Lambda$ and Weyl's dimension formula gives

$$
\mathrm{rk}_{\mathbb{Z}} M_{\Lambda}(m)=\operatorname{dim}_{\mathbb{R}}\left(V_{\Lambda}(m)\right)=\frac{m^{2}}{2}+O(m),
$$

as $m \rightarrow \infty$. Secondly, if $\Lambda=\tau_{1} \omega_{1}+\tau_{2} \omega_{2}, \tau_{1}, \tau_{2} \in \mathbb{N}, \tau_{1} \tau_{2} \neq 0$, then the condition $\Lambda \neq \Lambda_{\theta}$ is equivalent to $\tau_{1} \neq \tau_{2}$ and again by Weyl's dimension formula one has

$$
\operatorname{rk}_{\mathbb{Z}} M_{\Lambda}(m)=\operatorname{dim}_{\mathbb{R}}\left(V_{\Lambda}(m)\right)=\frac{\tau_{1}^{2} \tau_{1}+\tau_{2}^{2} \tau_{1}}{2} m^{3}+O\left(m^{2}\right)
$$

as $m \rightarrow \infty$. Let $M_{i, m}:=M_{\omega_{i}}(m), i=1,2$. Then it follows that

$$
\liminf _{m} \sum_{j=0}^{2} \frac{\log \left|H^{2 j+1}\left(\Gamma, M_{i, m}\right)\right|}{m^{3}} \geq \frac{2 \pi}{9 \operatorname{vol}\left(\widetilde{X}_{d}\right)} \operatorname{vol}(X) .
$$

Again, by (1.1) and (1.2), one is led to the following conjecture.

Conjecture 1.4. Let $\Gamma$ and $M_{i, m}, m \in \mathbb{N}$, be as above. Then one has

$$
\lim _{m \rightarrow \infty} \frac{\log \left|H^{3}\left(\Gamma, M_{i, m}\right)\right|}{m^{3}}=\frac{2 \pi}{9 \operatorname{vol}\left(\widetilde{X}_{d}\right)} \operatorname{vol}(X)
$$

and

$$
\log \left|H^{j}\left(\Gamma, M_{i, m}\right)\right|=o\left(m^{3}\right), \quad j \neq 3 .
$$

There are similar statements for highest weights $\Lambda=\tau_{1} \omega_{1}+\tau_{2} \omega_{2}$ with $\tau_{1} \tau_{2} \neq 0$.

Next we describe our approach to prove the main results. As in MaMü, it is based on the study of the analytic torsion. To begin with we consider an arbitrary connected semi-simple algebraic group $G$ over $\mathbb{Q}$. Let the notation be as at the beginning of the introduction. Assume that $\delta(\widetilde{X})=1$. Choose a $G$-invariant Riemannian metric $g$ on $\tilde{X}$. Assume that $\Gamma \subset G(\mathbb{Q})$ is torsion free. Let $X:=\Gamma \backslash \widetilde{X}$ equipped with the metric induced by $G$. Then we have $H^{*}(\Gamma, M)=H^{*}(X, \mathcal{M})$. Let $V:=M \otimes_{\mathbb{Z}} \mathbb{R}$ and let $\rho: G(\mathbb{R}) \rightarrow \operatorname{GL}(V)$ be the representation associated to the arithmetic $\Gamma$-module $M$. Let $E \rightarrow X$ be the flat 
vector bundle associated to $\left.\rho\right|_{\Gamma}$. Choose a Hermitian fibre metric in $E$. Let $T_{X}(\rho) \in \mathbb{R}^{+}$ be the analytic torsion of $X$ and $E$. Recall that

$$
\log T_{X}(\rho)=\left.\frac{1}{2} \sum_{p=1}^{n}(-1)^{p} p \frac{d}{d s} \zeta_{p}(s ; \rho)\right|_{s=0},
$$

where $\zeta_{p}(s ; \rho)$ is the zeta function of the Laplace operator $\Delta_{p}(\rho)$ on $E$-valued $p$-forms and $n=\operatorname{dim} X$ (see [M̈̈1]). Assume that $\rho$ is acyclic, that is $H^{*}(X, E)=0$. Then $T_{X}(\rho)$ is metric independent [Mü1, Corollary 2.7] and equals the Reidemeister torsion $\tau_{X}(\rho)$ [Mü1, Theorem 1]. Moreover, $H^{*}(\Gamma, M)$ is a finite abelian group. Using Proposition 2.1, we get

$$
\log T_{X}(\rho)=\sum_{q=0}^{n}(-1)^{q+1} \log \left|H^{q}(\Gamma, M)\right| .
$$

This is the key equality which we apply to prove Theorems 1.1 and 1.3 . In $\mathbb{M P}$ we studied the asymptotic behavior of $T_{X}\left(\tau_{m}\right)$ for certain sequences of irreducible representations of $G(\mathbb{R})^{0}$. We will apply the results of $[\mathbb{M P}]$ to our case. The main issue is to construct appropriate arithmetic $\Gamma$-modules.

We start with the case of $\widetilde{X}=\mathrm{SO}^{0}(p, q) / \mathrm{SO}(p) \times \mathrm{SO}(q), p, q$ odd. Let $G=\mathrm{SO}_{Q}$ be the special orthogonal group of a quadratic form $Q$ over a totlally real number field $F$ as defined above. Then $G$ is a connected algebraic group over $F$. Let $G^{\prime}=\operatorname{Res}_{F / \mathbb{Q}}(G)$ be the algebraic $\mathbb{Q}$-group obtained from $G$ by restriction of scalars [Wei]. The group of real points $G^{\prime}(\mathbb{R})$ is given by

$$
G^{\prime}(\mathbb{R}) \cong \mathrm{SO}(p, q) \times K_{1},
$$

where $K_{1}$ is the product of $d-1$ copies of $\mathrm{SO}(p+q)$. Then we construct a sequence $\rho(m): G^{\prime} \rightarrow G L\left(V_{m}\right), m \in \mathbb{N}$, of $\mathbb{Q}$-rational representations such that the irreducible components of $\rho(m)(\mathbb{R}): G^{\prime}(\mathbb{R})^{0} \rightarrow \mathrm{GL}\left(V_{m} \otimes_{\mathbb{Q}} \mathbb{R}\right)$ are of the form considered in $\mathbb{M P}$, Theorem 1.1]. Let $\Delta: G \rightarrow G^{\prime}$ be the diagonal embedding. Let $\Gamma^{\prime}=\Delta(\Gamma)$. Then $\Gamma^{\prime}$ is an arithmetic subgroup of $G^{\prime}(\mathbb{Q})$. Therefore, $V_{m}$ contains a lattice $M_{m}$ which is invariant under $\rho(m)\left(\Gamma^{\prime}\right)$. Through the isomorphism $\Gamma \cong \Gamma^{\prime}, M_{m}$ becomes a $\Gamma$-module. This is our arithmetic $\Gamma$-module. By construction we have $H^{*}\left(\Gamma, M_{m}\right) \cong H^{*}\left(\Gamma^{\prime}, M_{m}\right)$. Thus it suffices to prove the statement of Theorem 1.1 for $\Gamma^{\prime}$.

Let $K^{\prime}=\mathrm{SO}(p) \times \mathrm{SO}(q) \times K_{1}$. Then $K^{\prime}$ is a maximal compact subgroup of $G^{\prime}(\mathbb{R})^{0}$. Let $\tilde{X}^{\prime}=G^{\prime}(\mathbb{R})^{0} / K^{\prime}$ and $X^{\prime}:=\Gamma^{\prime} \backslash \widetilde{X}^{\prime}$. Now we apply $\mathbb{M P}$, Propositions $\left.1.2,1.3\right]$ to determine the asymptotic behavior of $T_{X^{\prime}}(\rho(m))$ as $m \rightarrow \infty$. Finally we use (1.10) to establish Theorem 1.1.

The proof of Theorem 1.3 uses similar arguments, which are also based on (1.10) and |MP.

The paper is organized as follows. In section 2 we collect some facts about cohomology of fundamental groups of manifolds with coefficients in a free $\mathbb{Z}$-module. We also recall some elementary facts about algebraic groups. In section 3 we consider arithmetic subgroups of $\mathrm{SO}^{0}(p, q)$ and prove Theorem 1.1. The prove of Theorem 1.3 is the content of the final section 1 . 


\section{PRELIMINARIES}

Let $X$ be a closed connected smooth manifold of dimension $d$. Let $\Gamma:=\pi_{1}\left(X, x_{0}\right)$ be the fundamental group of $X$ with respect to some base point $x_{0}$ and let $\widetilde{X}$ be the correpsonding universal covering. Thus $\Gamma$ acts properly discontinuously and fixed point free on $\widetilde{X}$ and $X=\Gamma \backslash \widetilde{X}$. Assume that $\tilde{X}$ is contractible.

Let $M$ be a free finite-rank $\mathbb{Z}$-module and let $\rho$ be a representation of $\Gamma$ on $M$. Let $H^{q}(\Gamma, M)$ be the $q$-th cohomology group of $\Gamma$ with coefficients in $M$, see $[\mathrm{Br}$. These groups can be computed as follows. Let $L$ be a smooth triangulation of $X$ and let $\widetilde{L}$ be the lift of $L$ to a triangulation of $\widetilde{X}$. Let $C_{q}(\widetilde{L} ; \mathbb{Z})$ be the free abelian group generated by the $q$-chains of $\widetilde{L}$, let $C^{q}(\widetilde{L} ; \mathbb{Z}):=\operatorname{Hom}_{\mathbb{Z}}\left(C_{q}(\widetilde{L}, \mathbb{Z}) ; \mathbb{Z}\right)$ and let $C_{*}(\widetilde{L} ; \mathbb{Z}) \operatorname{resp} . C^{*}(\widetilde{L} ; \mathbb{Z})$ be the associated simplical chain- resp. cochain complexes. Each $C_{q}(\widetilde{L} ; \mathbb{Z})$ is a free $\mathbb{Z}[\Gamma] \operatorname{module}$ and if one fixes an embedding of $L$ into $\widetilde{L}$, then the $q$-cells of $L$ form a base of $C_{q}(\widetilde{L} ; \mathbb{Z})$ over $\mathbb{Z}[\Gamma]$. Let

$$
C^{q}(L, M):=C^{q}(\widetilde{L} ; \mathbb{Z}) \otimes_{\mathbb{Z}[\Gamma]} M .
$$

Then the $C^{q}(L, M)$ form again a cochain complex $C^{*}(L, M)$ and the corresponding cohomology groups will be denoted by $H^{q}(L, M)$. There is an isomorphism

$$
C^{q}(L, M) \cong \operatorname{Hom}_{\mathbb{Z}[\Gamma]}\left(C_{q}(\widetilde{L} ; \mathbb{Z}), M\right)
$$

which induces an isomorphism of the corresponding cochain complexes. Since $\widetilde{L}$ is contractible, the complex $C_{*}(\widetilde{L})$ is a free resolution of $\mathbb{Z}$ over $\mathbb{Z}[\Gamma]$ and thus one has

$$
H^{q}(\Gamma, M) \cong H^{q}\left(\operatorname{Hom}_{\mathbb{Z}[\Gamma]}\left(C_{*}(\widetilde{L}), M\right)\right) \cong H^{q}(L, M) .
$$

Each cohomology group $H^{q}(L, M)$ is a finitely generated abelian group. Let $H^{q}(\Gamma, M)_{\text {tors }}$ be the torsion subgroup of $H^{q}(\Gamma, M)$ and let $H^{q}(\Gamma, M)_{\text {free }}=H^{q}(\Gamma, M) / H^{q}(\Gamma, M)_{\text {tors }}$ be the free part. Then one has

$$
H^{q}(\Gamma, M)=H^{q}(\Gamma, M)_{\text {free }} \oplus H^{q}(\Gamma, M)_{\text {tors }} .
$$

Now let $V:=M \otimes_{\mathbb{Z}} \mathbb{C}$ and $V_{\mathbb{R}}:=M \otimes_{\mathbb{Z}} \mathbb{R}$. Then $V$ is a finite-dimensional complex vector space, $V_{\mathbb{R}} \subset V$ is a real structure on $V$ and $M$ is a lattice in $V_{\mathbb{R}}$. We regard $\rho$ as a representation of $\Gamma$ on $V$. Then $\rho$ is unimodular, i.e., $|\operatorname{det} \rho(\gamma)|=1$ for all $\gamma \in \Gamma$. Let

$$
C^{q}(L, V):=C^{q}(\widetilde{L}) \otimes_{\mathbb{Z}[\Gamma]} V .
$$

The $C^{q}(L, V)$ 's form a chain complex $C^{*}(L, V)$ of finite-dimensional $\mathbb{C}$-vector spaces and one has

$$
C^{q}(L, V)=C^{q}(L, M) \otimes_{\mathbb{Z}} \mathbb{C} .
$$

Let $E:=\tilde{X} \times{ }_{\rho} V$ be the flat vector bundle over $X$ associated to $\rho$. Then by the de Rham isomorphism, the cohomology groups $H^{q}(L, V)$ of the complex $C^{*}(L, V)$ are canonically isomorphic to the cohomology groups $H^{q}(X, E)$ of the complex of $E$-valued differential forms on $X$. By Hodge theory they are canonically isomorphic to the space of $E$-valued harmonic forms for any choice of metrics on $X$ and $E$, respectively. 
We assume that the bundle $E$ is acyclic i.e. that $H^{q}(L ; V)=H^{q}(X ; E)=0$ for all $q$. This holds in all cases that we study in this paper. Let $\sigma_{j}^{q}, j=1, \ldots, r_{q}$, be the oriented $q$-simplices of $L$ considered as a preferred basis of the $\mathbb{Z}[\Gamma]$-module $C^{q}(\widetilde{L} ; \mathbb{Z})$. Let $e_{1}, \ldots, e_{m}$ be a basis of $M$. Then $\left\{\sigma_{j}^{q} \otimes e_{k}: j=1, \ldots, r_{q}, k=1, \ldots, m\right\}$ is a preferred basis of $C^{q}(L ; M)$ and also of $C^{q}(L ; V)$. Let $\tau_{X}(\rho) \in \mathbb{R}^{+}$be the Reidemeister torsion with respect to these volume elements (see $\mathbb{M u ̈ 1}$, $\mathbb{M a M u ̈} \mid$ ). Note that $\tau_{X}(\rho)=\left|\tau_{X}^{\mathbb{C}}(\rho)\right|$, where $\tau_{X}^{\mathbb{C}}(\rho) \in \mathbb{C}^{\times}$is the complex Reidemeister torsion. Since $\rho$ is acyclic, $\tau_{X}(\rho)$ is a combinatorial invariant of $X$ and $\rho$ which is independent of the choices that we made (see [Mü1, section $1])$. Moreover, each cohomology group $H^{q}(\Gamma, M)$ is finite, i.e., $H^{q}(\Gamma, M)=H^{q}(\Gamma, M)_{\text {tors }}$ and the order $\left|H^{q}(\Gamma, M)\right|$ of these groups is related to the Reidemeister torsion as follows.

Proposition 2.1. Assume that $H^{q}(X, E)=0$ for all $q$. Then one has

$$
\sum_{q=0}^{d}(-1)^{q+1} \log \left|H^{q}(\Gamma, M)\right|=\log \tau_{X}(\rho) .
$$

Proof. Let $C^{*}\left(L, V_{\mathbb{R}}\right)$ be the chain complex of the finite-dimensional real vector spaces

$$
C^{q}\left(L, V_{\mathbb{R}}\right):=C^{q}(\widetilde{L}) \otimes_{\mathbb{Z}[\Gamma]} V_{\mathbb{R}}, \quad q=0, \ldots, d .
$$

We have

$$
C^{q}\left(L, V_{\mathbb{R}}\right)=C^{q}(L, M) \otimes_{\mathbb{Z}} \mathbb{R} .
$$

Let $\rho_{\mathbb{R}}: \Gamma \rightarrow \operatorname{GL}\left(V_{\mathbb{R}}\right)$ be the representation induced by $\rho$ and let $E_{\mathbb{R}}:=\widetilde{X} \times_{\rho_{\mathbb{R}}} V_{\mathbb{R}}$ be the associated flat real vector bundle. Then $H^{*}\left(X ; E_{\mathbb{R}}\right)=0$. The basis of the free $\mathbb{Z}$-module $C^{q}(L ; M)$, described above, gives rise to a distinguished basis of $C^{q}\left(L ; V_{\mathbb{R}}\right)$. Let $\tau_{X}\left(\rho_{\mathbb{R}}\right)$ be the Reidemeister torsion of the complex $C^{*}\left(L ; V_{\mathbb{R}}\right)$ with respect to volume elements defined by these bases. Then it follows from (2.11) and (2.12) as in [BV], section 2.2] that

$$
\log \tau_{X}\left(\rho_{\mathbb{R}}\right)=\sum_{q=0}^{d}(-1)^{q+1} \log \left|H^{q}(\Gamma, M)\right| .
$$

See also MaMü, Proposition 2.3] and [Tu, Lemma 2.1.1]. Since the coboundary operators of the complexes $C^{*}\left(L ; V_{\mathbb{R}}\right)$ and $C^{*}(L ; V)$, respectively, are induced by the coboundary operators of $C^{*}(L ; M)$, it follows from the definition of the Reidemeister torsion that $\tau_{X}\left(\rho_{\mathbb{R}}\right)=\tau_{X}(\rho)$. Combined with (2.13) the proposition follows.

Finally we recall some facts conerning linear algebraic groups. For all details we refer to [Bo2]. Let $F$ be a finite Galois extension of $\mathbb{Q}$ with Galois group $\Sigma:=\operatorname{Gal}(F / \mathbb{Q})$. For $\sigma \in \Sigma$ and $x \in F$ let $x^{\sigma}$ denote the image of $x$ under $\sigma$. If $G$ is a linear algebraic group over $F$ with coordinate algebra $F[G]$, let $G^{\sigma}$ denote the linear algebraic group conjugate by $\sigma$, see [Bo2]. If $G$ is realized as the zero set in some $F^{n}$ of an ideal $I$ in $F\left[X_{1}, \ldots, X_{n}\right]$, then $G^{\sigma}$ is the zero set of the ideal $I^{\sigma}$, where $I^{\sigma}$ is obtained from $I$ by applying $\sigma$ to each polynomial coefficient. Each $F$-rational homomorphism $\rho: G \rightarrow H$ of linear algebraic groups over $F$ induces canonically an $F$-rational homomorphism $\rho^{\sigma}: G^{\sigma} \rightarrow H^{\sigma}$. 
If $G$ is an algebraic group defined over $F$, an algebraic group $G^{\prime}$ defined over $\mathbb{Q}$ together with an $F$-rational isomorphism $\mu: G^{\prime} \times_{\mathbb{Q}} F \rightarrow G$ is called a $\mathbb{Q}$-structure of $G$. The $\mathbb{Q}$ structure canonically induces an action of $\Sigma$ on the coordinate algebra of $G$ and thus on $G$ itself.

Let $V$ be a finite-dimensional $F$-vector space. A $\mathbb{Q}$-structure $V_{0}$ of $V$ is a $\mathbb{Q}$-subspace $V_{0}$ of $V$ such that the embedding $V_{0} \hookrightarrow V$ induces an isomorphism $V_{0} \otimes_{\mathbb{Q}} F$ of $F$-vector spaces. For $\sigma \in \Sigma$ a $\mathbb{Q}$-linear automorphism $A$ of $V$ is called $\sigma$-linear if $A(\lambda v)=\sigma(\lambda) A(v)$, $\lambda \in F, v \in V$. Then a semi-linear action of $\Sigma$ on $V$ is given by a family $\left\{f_{\sigma}\right\}_{\sigma \in \Sigma}$ of $\sigma$-linear automorphisms $f_{\sigma}$ of $V$, satisfying $f_{\sigma \tau}=f_{\sigma} \circ f_{\tau}, \sigma, \tau \in \Sigma$. Given a semi-linear action of $\Sigma$ on $V$, the set $V^{\Sigma}:=\left\{v \in V: f_{\sigma}(v)=v, \forall \sigma \in \Sigma\right\}$ is a $\mathbb{Q}$-structure of $V$ and every $\mathbb{Q}$-structure is of this form (see $\mathbb{B o 2}$, AG.14.2]). If $V_{0}$ is a $\mathbb{Q}$-structure of $V$, then $\mathrm{GL}\left(V_{0}\right)$ is a $\mathbb{Q}$-structure of $\mathrm{GL}(V)$ and the corresponding action of $\Sigma$ on $\mathrm{GL}(V)$ is given by $\sigma \cdot g:=f_{\sigma} \circ g \circ f_{\sigma}^{-1}, g \in \mathrm{GL}(V)$.

\section{Arithmetic subgroups of $\operatorname{SO}^{0}(p, q)$}

Let $p, q \in \mathbb{N}$ be odd. Put

$$
p_{1}=(p-1) / 2, q_{1}=(q-1) / 2, n:=p_{1}+q_{1} .
$$

We denote by $\mathrm{SO}(p, q)$ the group of isometries of the standard quadratic form of signature $(p, q)$ on $\mathbb{R}^{p+q}$ with determinant 1 . Let $\mathrm{SO}^{0}(p, q)$ denote the identity component of $\operatorname{SO}(p, q)$. The group $\mathrm{SO}^{0}(p, q)$ is of fundamental rank one. Let $\mathfrak{g}$ be the Lie algebra of $\mathrm{SO}^{0}(p, q)$. We choose the fundamental Cartan subalgebra as follows. Let $E_{i, j}$ be the $(p+q) \times(p+q)$-matrix which is one at the $i$-th row and $j$-th column and which is zero elsewhere. Let

$$
H_{1}:=E_{p, p+1}+E_{p+1, p} .
$$

and let

$$
H_{i}:= \begin{cases}\sqrt{-1}\left(E_{2 i-3,2 i-2}-E_{2 i-2,2 i-3}\right), & 2 \leq i \leq p_{1}+1 \\ \sqrt{-1}\left(E_{2 i-1,2 i}-E_{2 i, 2 i-1}\right) & p_{1}+1<i \leq n+1\end{cases}
$$

Then

$$
\mathfrak{h}:=H_{1} \oplus \bigoplus_{i=2}^{n+1} \sqrt{-1} H_{i}
$$

is a Cartan subalgebra of $\mathfrak{g}$. Define $e_{i} \in \mathfrak{h}_{\mathbb{C}}^{*}, i=1, \ldots, n+1$, by

$$
e_{i}\left(H_{j}\right)=\delta_{i, j}, 1 \leq i, j \leq n+1 \text {. }
$$

The finite-dimensional irreducible complex representations $\tau$ of $\operatorname{SO}^{0}(p, q)$ are parametrized by their highest weights $\Lambda(\tau) \in \mathfrak{h}_{\mathbb{C}}^{*}$ given by

$$
\begin{aligned}
\Lambda(\tau)= & k_{1}(\tau) e_{1}+\cdots+k_{n+1}(\tau) e_{n+1}, \quad\left(k_{1}(\tau), \ldots k_{n+1}(\tau)\right) \in \mathbb{Z}^{n+1}, \\
& k_{1}(\tau) \geq k_{2}(\tau) \geq \cdots \geq k_{n}(\tau) \geq\left|k_{n+1}(\tau)\right| .
\end{aligned}
$$


For $\Lambda(\tau)$ a weight as in (3.16), the highest weight $\Lambda\left(\tau_{\theta}\right)$ of the representation $\tau \circ \theta$ is

$$
\Lambda\left(\tau_{\theta}\right)=k_{1}(\tau) e_{1}+\cdots+k_{n}(\tau) e_{n}-k_{n+1}(\tau) e_{n+1} .
$$

If we let

$$
\omega_{f, n}^{+}:=\sum_{j=1}^{n+1} e_{j} ; \quad \omega_{f, n}^{-}:=\left(\omega_{f, n}^{+}\right)_{\theta}=\sum_{j=1}^{n} e_{j}-e_{n+1},
$$

then $\frac{1}{2} \omega_{f, n}^{ \pm}$are the fundamental weights which are not invariant under $\theta$. We now recall the construction of certain arithmetically defined cocompact subgroups of $\operatorname{SO}^{0}(p, q)$. For more details see [Sch, section 3.2, Appendix B] and for the $\operatorname{SO}^{0}(p, 1)$-case [Mil]].

Let $F$ be a totally real number field of degree $d=[F: \mathbb{Q}]>1$. Let $\Sigma$ be the Galois group of $F$ over $\mathbb{Q}$. We fix an embedding $F \subset \mathbb{R}$. Let $1 \in \Sigma$ be the identity. Let $\alpha_{j} \in F^{*}$, $j=1, \ldots, p+q$, be such that

$$
\operatorname{sign}\left(\alpha_{j}\right)= \begin{cases}+1, & \text { if } j \leq p \\ -1, & \text { if } p<j \leq p+q\end{cases}
$$

and

$$
\operatorname{sign}\left(\sigma\left(\alpha_{j}\right)\right)=+1, \quad \sigma \in \Sigma \backslash\{1\}, j=1, \ldots, p+q .
$$

For $\sigma \in \Sigma$ let $Q^{\sigma}$ be the quadratic form on $\mathbb{R}^{p+q}$ defined by

$$
Q^{\sigma}(x)=\sum_{j=1}^{p+q} \sigma\left(\alpha_{j}\right) x_{j}^{2} .
$$

Then $Q:=Q^{1}$ is a non-degenerate quadratic form of signature $(p, q)$ and $Q^{\sigma}, \sigma \neq 1$, is positive definite.

Let $G:=\mathrm{SO}_{Q} \subset \mathrm{GL}_{p+q}$ be the special orthogonal group of $Q$, i.e., the subgroup of all elements of determinant one leaving $Q$ invariant. Then $G$ is a connected algebraic group defined over $F$. Let $J \in \mathrm{GL}_{p+q}(\mathbb{R})$ be defined by

$$
J:=\operatorname{diag}\left(\sqrt{\alpha_{1}}, \ldots, \sqrt{\alpha_{p}}, \sqrt{-\alpha_{p+1}}, \ldots, \sqrt{-\alpha_{p+q}}\right) .
$$

Then the map $g \mapsto J g J^{-1}$ establishes an isomorphism $G(\mathbb{R}) \cong \operatorname{SO}(p, q)$. Similarly, we have $G^{\sigma}(\mathbb{R}) \cong \mathrm{SO}(p+q)$, if $\sigma \neq 1$. Let

$$
G^{\prime} \cong \operatorname{Res}_{F / \mathbb{Q}}(G)
$$

be the algebraic $\mathbb{Q}$-group obtained by restriction of scalars. There is a canonical isomorphism of algebraic groups over $F$

$$
\alpha: G^{\prime} \times_{\mathbb{Q}} F \cong \prod_{\sigma \in \Sigma} G^{\sigma},
$$

and the group of real points $G^{\prime}(\mathbb{R})$ satisfies

$$
G^{\prime}(\mathbb{R}) \cong \mathrm{SO}(p, q) \times \prod_{\sigma \in \Sigma \backslash\{1\}} \mathrm{SO}(p+q) .
$$


Denote by

$$
\Delta: G \rightarrow \prod_{\sigma \in \Sigma} G^{\sigma}
$$

the diagonal embedding given by $\Delta(g)=\left(g^{\sigma}\right)_{\sigma \in \Sigma}$.

Let $\mathcal{O}_{F}$ be the ring of integers of $F$ and let $G_{\mathcal{O}_{F}}$ be the group of $\mathcal{O}_{F}$-units of $G$. An arithmetic subgroups of $G(F)$ is by definition a subgroup which is commensurable with $G_{\mathcal{O}_{F}}$. Let $\Gamma_{0}:=J G_{\mathcal{O}_{F}} J^{-1}$. Then $\Gamma_{0}$ is a subgroup of $\operatorname{SO}(p, q)$.

Lemma 3.1. $\Gamma_{0}$ is a discrete, cocompact subgroup of $\mathrm{SO}(p, q)$.

Proof. For $\sigma \in \Sigma \backslash\{1\}$, the group $G^{\sigma}(\mathbb{R})$ is isomorphic to $\mathrm{SO}(p+q)$. Thus by BoHa, p. 530], $\Gamma_{0}$ is discrete in $\mathrm{SO}(p, q)$. Since all quadratic forms $Q^{\sigma}, \sigma \neq 1$, are positive definite, the form $Q$ is anisotropic over $F$. Thus, by [Bo2, page 256] $G$ is anisotropic over $F$. Therefore, $G_{\mathcal{O}_{F}}$ contains no non-trivial unipotent elements. Using [BoHa, Lemma 11.4, Theorem 12.3], it follows that the diagonal image of $G_{\mathcal{O}_{F}}$ in $\prod_{\sigma \in \Sigma} G^{\sigma}(\mathbb{R})$ is cocompact and since $G^{\sigma}(\mathbb{R})$ is compact for $\sigma \neq 1, \Gamma_{0}$ is also cocompact in $\operatorname{SO}(p, q)$.

Remark 3.2. If $F=Q[\sqrt{v}]$ is a real quadratic field, then putting $\alpha_{1}=\cdots=\alpha_{p}=1$, $\alpha_{p+1}=\cdots=\alpha_{p+q}=-\sqrt{v}$ the above construction has already been given in [Bo1, section $4.3]$.

Now we let $B$ be the symmetric bilinear form on $F^{p+q}$ given by

$$
B\left(e_{i}, e_{j}\right)=\left\{\begin{array}{ll}
1, & i+j=p+q+1 \\
0, & i+j \neq p+q+1
\end{array},\right.
$$

for $e_{1}, \ldots, e_{p+q}$ the standard base of $F^{p+q}$. Let $\mathrm{O}_{B}$ be the orthogonal group of $B$ and let $\mathrm{SO}_{B}$ be the elements of $\mathrm{O}_{B}$ of determinant one. Then $\mathrm{O}_{B}$ and $\mathrm{SO}_{B}$ are algebraic groups defined over $F$ and there exists an isomorphism $\mu: G(\bar{F}) \rightarrow \mathrm{SO}_{B}(\bar{F})$, i.e. $G$ is a form of $\mathrm{SO}_{B}$ over $F$.

Let $T$ be the maximal torus of $\mathrm{SO}_{B}(\bar{F})$ given by

$$
T=\left\{\operatorname{diag}\left(\lambda_{1}, \ldots, \lambda_{n+1}, \lambda_{1}^{-1}, \ldots, \lambda_{n+1}^{-1}\right), \lambda_{1}, \ldots, \lambda_{n+1} \in \bar{F}^{*}\right\},
$$

where $n=(p+q) / 2-1$. Then $T$ is defined over $F$. Let $X(T)$ be the character group of $T$, written additively. Then a base of $X(T)$ is given by the $f_{i}: T \rightarrow \bar{F}$, $f_{i}\left(\operatorname{diag}\left(\lambda_{1}, \ldots, \lambda_{n+1}, \lambda_{1}^{-1}, \ldots, \lambda_{n+1}^{-1}\right)\right)=\lambda_{i}$, where $1 \leq i \leq n+1$.

By $\operatorname{Rep}\left(\mathrm{SO}_{B}(\bar{F})\right)$ we denote the finite-dimensional representations of $\mathrm{SO}_{B}(\bar{F})$ which are irreducible. Then the elements of $\operatorname{Rep}\left(\mathrm{SO}_{B}(\bar{F})\right)$ correspond bijectively to their highest weights $\lambda_{\tau}:=m_{1} f_{1}+\cdots+m_{n+1} f_{n+1}$, where $m_{1}, \ldots, m_{n+1} \in \mathbb{Z}, m_{1} \geq m_{2} \geq \cdots \geq$ $m_{n} \geq\left|m_{n+1}\right|$. Since $T$ is split over $F$, every finite-dimensional irreducible representation of $\mathrm{SO}_{B}(\bar{F})$ is defined over $F$, [Til, Proposition 2.3].

For $\tau \in \operatorname{Rep}\left(\mathrm{SO}_{B}(\bar{F})\right)$ with highest weight $\lambda_{\tau}=m_{1}(\tau) f_{1}+\cdots+m_{n+1}(\tau) f_{n+1}$ let $\tau^{\prime} \in$ $\operatorname{Rep}\left(\mathrm{SO}_{B}(\bar{F})\right)$ be the element with highest weight $\lambda_{\tau^{\prime}}=m_{1}(\tau) f_{1}+\cdots+m_{n} f_{n}-m_{n+1}(\tau) f_{n+1}$. Then the following lemma holds. 
Lemma 3.3. For every $\tau \in \operatorname{Rep}\left(\mathrm{SO}_{B}(\bar{F})\right)$ there exists a representation $\tilde{\tau}$ of $\mathrm{O}_{B}(\bar{F})$ that restricts to $\tau+\tau^{\prime}$ on $\mathrm{SO}_{B}(\bar{F})$.

Proof. The proof of the corresponding proposition for $\mathrm{SO}_{B}(\mathbb{C})$ given in [GW], Theorem 5.22 ] extends withouth difficulty to any algebraically closed field of characteristic zero.

Remark 3.4. If $\tau$ satisfies $\tau=\tau^{\prime}$ then there exists in fact a representation of $\mathrm{O}_{B}$ that restricts to $\tau$. However, we are only interested in the case $\tau \neq \tau^{\prime}$ and in this case the representation $\tilde{\tau}$ from the previous lemma is irreducible.

Now we let $\mathrm{PSO}_{B}:=\mathrm{SO}_{B} /\{ \pm \mathrm{Id}\}$. Then an element $\tau \in \operatorname{Rep}\left(\mathrm{SO}_{B}(\bar{F})\right)$ of highest weight $\lambda_{\tau}=m_{1} f_{1}+\cdots+m_{n+1} f_{n}$ descends to a representation of $\operatorname{PSO}_{B}(\bar{F})$ if and only $m_{1}+\cdots+m_{n+1}$ is even.

Lemma 3.5. Let $\tau$ be a representation of $\mathrm{SO}_{B}$ over $\bar{F}$ which descends to a representation of $\mathrm{PSO}_{B}$ over $\bar{F}$. Then there exists an $F$-rational representation of $G$ which over $\bar{F}$ is equivalent to $\left(\tau+\tau^{\prime}\right) \circ \mu$.

Proof. For $\sigma \in \operatorname{Gal}(\bar{F} / F)$ define an automorphism $\phi_{\sigma}$ of $\operatorname{SO}_{B}(\bar{F})$ by $\phi_{\sigma}:=\mu \circ \sigma \circ \mu^{-1} \circ$ $\sigma^{-1}$. Since the Dynkin diagram $D_{n+1}$ has exactly one non-trivial automorphism, there is a natural isomorphism $\operatorname{Aut}\left(\mathrm{SO}_{B}(\bar{F})\right) \cong \mathrm{PO}_{B}(\bar{F})$, where $\mathrm{PO}_{B}(\bar{F})$ acts on $\mathrm{SO}_{B}(\bar{F})$ by conjugation, and thus there exists a unique $a_{\sigma} \in \mathrm{PO}_{B}(\bar{F})$ such that for each $g \in \mathrm{SO}_{B}(\bar{F})$ one has $\phi_{\sigma}(g)=a_{\sigma} g a_{\sigma}^{-1}$. Thus one has

$$
\mu(\sigma(g))=a_{\sigma} \sigma(\mu(g)) a_{\sigma}^{-1} .
$$

We can regard the assignment $\sigma \rightarrow a_{\sigma}$ as an element of the first Galois-cohomology set $H^{1}\left(\operatorname{Gal}(\bar{F} / F), \mathrm{PO}_{B}(\bar{F})\right)$. By Lemma 3.3 there exist a representation $\tilde{\tau}$ of $\mathrm{PO}_{B}(\bar{F})$ on $V_{\tilde{\tau}}=V_{\tau} \oplus V_{\tau^{\prime}}$ which restricts to $\tau \oplus \tau^{\prime}$ on $\operatorname{SO}_{B}(\bar{F})$. The assignment $\sigma \mapsto \tilde{\tau}\left(a_{\sigma}\right)$ is an element of $H^{1}\left(\operatorname{Gal}(\bar{F} / F), \operatorname{GL}\left(V_{\tilde{\tau}}\right)\right)$ and since this set is trivial by Hilbert's theorem 90, there exists an $x \in \mathrm{GL}\left(V_{\tilde{\tau}}\right)$ such that

$$
\tilde{\tau}\left(a_{\sigma}\right)=x^{-1} \sigma(x) \quad \forall \sigma \in \operatorname{Gal}(\bar{F} / F) .
$$

Now define a representation $\rho$ of $G(\bar{F})$ by $\rho:=\operatorname{Int}(x) \circ\left(\tau+\tau^{\prime}\right) \circ \mu$. Then $\rho$ is equivalent to $\left(\tau+\tau^{\prime}\right) \circ \mu$. Applying (3.22) and (3.23) it follows that for $\sigma \in \operatorname{Gal}(\bar{F} / F)$ and $g \in G(\bar{F})$ one has

$$
\rho(\sigma(g))=x \tilde{\tau}\left(a_{\sigma}\right)\left(\tau+\tau^{\prime}\right)(\sigma(\mu(g))) \tilde{\tau}\left(a_{\sigma}^{-1}\right) x^{-1}=\sigma(x) \sigma\left(\left(\tau+\tau^{\prime}\right)(\mu(g))\right) \sigma(x)^{-1}=\sigma(\rho(g)),
$$

where we used that $\tau+\tau^{\prime}$ is defined over $F$ and hence commutes with $\operatorname{Gal}(\bar{F} / F)$. Thus $\rho$ commutes with $\operatorname{Gal}(\bar{F} / F)$ and thus it is defined over $F$.

Now we may fix an embedding of $\mathrm{SO}^{0}(p, q)$ into $\mathrm{SO}_{B}(\mathbb{C})$ such that the representations of $\mathrm{SO}^{0}(p, q)$ with highest weight $m_{1} e_{1}+\cdots+m_{n+1} e_{n+1}$ are the restrictions to $\mathrm{SO}^{0}(p, q)$ of the representation of $\mathrm{SO}_{B}(\mathbb{C})$ with highest weight $m_{1} f_{1}+\cdots+m_{n+1} f_{n+1}$.

The following proposition is certainly well known and was used already by Bergeron and Venkatesh [BV, section 8.1]. However, for the sake of completeness we include a proof here. If $V$ is a finite-dimensional $F$-vector space, let $V^{\sigma}$ be the $F$-vector space with scalar-multiplication $a \cdot v:=\sigma(a) v, a \in F, v \in V$. 
Lemma 3.6. Let $G^{\prime}$ be an algebraic group defined over $\mathbb{Q}$. Let $V$ be a finite-dimensional $F$-vector space and let $\rho: G^{\prime} \rightarrow \mathrm{GL}(V)$ be a representation defined over $F$. Then $\tilde{\rho}:=$ $\prod_{\sigma \in \Sigma} \rho^{\sigma^{-1}}$ is defined over $\mathbb{Q}$, where $\rho^{\sigma^{-1}}$ is regarded as an $F$-rational representation of $G^{\prime}$ on $V^{\sigma}$.

Proof. Each $\sigma \in \Sigma$ acts on $\prod_{\sigma \in \Sigma} V^{\sigma}$ as a $\sigma$-linear automorphism by permuting the factors. The corresponding $\mathbb{Q}$-structure of $\prod_{\sigma \in \Sigma} V^{\sigma}$ is $V$, regarded as a $\mathbb{Q}$-vector space and embedded diagonally into $\prod_{\sigma \in \Sigma} V^{\sigma}$. Now it is easy to see that $\tilde{\rho}$ commutes with the action of $\Sigma$ on $G^{\prime}$ and the action of $\Sigma$ on $\operatorname{GL}\left(\prod_{\sigma \in \Sigma} V^{\sigma}\right)$ associated to this $\mathbb{Q}$-structure. Thus $\tilde{\rho}$ is defined over $\mathbb{Q}$.

Let $G^{\prime}(\mathbb{R})^{0}$ be the connected component of $1 \in G^{\prime}(\mathbb{R})$ and $G^{\prime}(\mathbb{R})^{c}:=\prod_{\sigma \in \Sigma \backslash\{1\}} \mathrm{SO}(p+q)$. Then we have

$$
G^{\prime}(\mathbb{R})^{0} \cong \operatorname{SO}^{0}(p, q) \times G^{\prime}(\mathbb{R})^{c} .
$$

Let $\theta$ be the standard Cartan-involution of $\operatorname{SO}^{0}(p, q)$. Then $\theta \otimes \operatorname{Id}_{G^{\prime}(\mathbb{R})^{c}}$ is a Cartan involution of $G^{\prime}(\mathbb{R})^{0}$ which we continue to denote by $\theta$. By $\operatorname{Rep}\left(G^{\prime}(\mathbb{R})^{0}\right)$ we denote the finite-dimensional irreducible complex representations of $G^{\prime}(\mathbb{R})^{0}$. For $\tau \in \operatorname{Rep}\left(G^{\prime}(\mathbb{R})^{0}\right)$, let $\tau_{\theta}$ be the element of $\operatorname{Rep}\left(G^{\prime}(\mathbb{R})^{0}\right)$ defined by $\tau_{\theta}:=\tau \circ \theta$.

Proposition 3.7. There exists a sequence $\rho(m)$ of $\mathbb{Q}$-rational representations of $G^{\prime}$ on finite-dimensional $\mathbb{Q}$-vector spaces $V_{\rho(m)}$ such that

(1) For the decomposition

$$
\rho(m)=\bigoplus_{\tau \in \operatorname{Rep}\left(G^{\prime}(\mathbb{R})^{0}\right)}[\rho(m): \tau] \tau,
$$

$[\rho(m): \tau] \in \mathbb{N}^{0}$ of $\rho(m)$, regarded as a complex representation of $G^{\prime}(\mathbb{R})^{0}$ on the vector space $V_{\rho(m)} \otimes_{\mathbb{Q}} \mathbb{C}$, into irreducible representations of $G^{\prime}(\mathbb{R})^{0}$ one has $\tau \neq \tau_{\theta}$ for each $\tau \in \operatorname{Rep}\left(G^{\prime}(\mathbb{R})^{0}\right)$ with $[\rho(m): \tau] \neq 0$.

(2) The dimension $\operatorname{dim}\left(V_{\rho(m)}\right)$ is a polynomial in $m$ with leading term

$$
\operatorname{dim}\left(V_{\rho(m)}\right)=C d m^{d n(n+1) / 2}+O\left(m^{d(n+1) / 2-1}\right),
$$

as $m \rightarrow \infty$, where $C>0$ is a constanst which depends only on $n$.

Proof. The Galois group $\Sigma$ acts on $\prod_{\sigma \in \Sigma} G^{\sigma}$ as follows. For $g \in \prod_{\sigma \in \Sigma} G^{\sigma}$ and $\sigma \in \Sigma$ we denote the projection of $g$ to $G^{\sigma}$ by $g_{\sigma}$. Then for $\sigma, \sigma^{\prime} \in \Sigma$ one has

$$
(\sigma g)_{\sigma^{\prime}}=\sigma\left(g_{\sigma^{-1} \sigma^{\prime}}\right)
$$

Now assume that for each $\sigma \in \Sigma$ we are given a finite-dimensional $F$-vector space $V_{\rho(\sigma)}$ and a representation $\rho(\sigma)$ of $G^{\sigma}$ on $V_{\rho(\sigma)}$, defined over $F$. Then the tensor-product

$$
\rho:=\bigotimes_{\sigma \in \Sigma} \rho(\sigma)
$$

is a representation of $\prod_{\sigma \in \Sigma} G^{\sigma}$ on $\bigotimes_{\sigma \in \Sigma} V_{\rho(\sigma)}$ and it follows that for $\sigma^{\prime} \in \Sigma$ one has

$$
\rho^{\sigma^{\prime}}=\bigotimes_{\sigma \in \Sigma} \rho\left(\sigma^{\prime-1} \sigma\right)^{\sigma^{\prime}} \text {. }
$$


Now if $n$ is even, for $m \in \mathbb{N}$ we let $\tau(m)$ be the representation of $G$ over $\bar{F}$ of highest weight $2 m e_{1}+\cdots+2 m e_{n+1}$. If $n$ is odd, we let $\tau(m)$ be the representation of highest weight $m e_{1}+\cdots+m e_{n+1}$. Then $\tau(m)$ and $\tau(m)_{\theta}$ descend to representations of $P G$. Thus by Lemma 3.5 there exists a representation of $G$ over $F$ which over $\bar{F}$ is equivalent to $\tau(m)+\tau(m)_{\theta}$. Thus if we define $\rho_{0}(m)$ by

$$
\rho_{0}(m):=\bigotimes_{\sigma \in \Sigma}\left(\tau(m)+\tau(m)_{\theta}\right)^{\sigma}
$$

then $\rho_{0}(m)$ is defined over $F$ and by (3.25), $\rho_{0}(m)$ is equivalent to $\rho_{0}(m)^{\sigma}$ for each $\sigma \in \Sigma$. Hence if we let $\rho(m)$ be the direct sum of $d$ copies of $\rho_{0}(m)$ then by Lemma $3.6 \rho(m)$ is defined over $\mathbb{Q}$. Each irreducible component of $\left.\rho(m)\right|_{G^{\prime}(\mathbb{R})^{0}}$, regarded as a complex representation of $G^{\prime}(\mathbb{R})^{0}$ on $V_{\rho(m)} \otimes_{\mathbb{Q}} \mathbb{C}$, is of the form $\tau(m) \otimes \pi$, or $\tau(m)_{\theta} \otimes \pi^{\prime}$, where $\pi$ and $\pi^{\prime}$ are irreducible representations of $G^{\prime}(\mathbb{R})^{c}$. Since $\tau(m)$ and $\tau(m)_{\theta}$ are not $\theta$-invariant, the same holds for each irreducible component of $\left.\rho(m)\right|_{G^{\prime}(\mathbb{R})^{0}}$. This proves the first statement. The second statement follows from Weyl's dimension formula.

We can now turn to the proof of Theorem 1.1. Let $\Delta$ be the diagonal embedding of $G$ into $\prod_{\sigma \in \Sigma} G^{\sigma}$. Then we can choose the isomorphism $\alpha$ in $(3.20)$ such that $\alpha\left(G_{\mathbb{Z}}^{\prime}\right)=\Delta\left(G_{\mathcal{O}_{F}}\right)$. Let $\Gamma \subset G_{\mathcal{O}_{F}}$ be a subgroup of finite index. Via the isomorphism $G(\mathbb{R}) \cong \mathrm{SO}(p, q)$ we identify $\Gamma$ with a subgroup of $\mathrm{SO}(p, q)$. We choose $\Gamma$ such that it is torsion free and is contained in $\mathrm{SO}^{0}(p, q)$. By Lemma 3.1, $\Gamma$ is a cocompact lattice in $\mathrm{SO}^{0}(p, q)$. Let $\Gamma^{\prime}=\Delta(\Gamma)$. Since $\Gamma$ and $\Gamma^{\prime}$ are isomorphic, it suffices to prove the statements of Theorem 1.1 for $\Gamma^{\prime}$.

The group $K_{0}:=\mathrm{SO}(p) \times \mathrm{SO}(q)$ is a a maximal compact subgroup of $\mathrm{SO}^{0}(p, q)$. Put

$$
K^{\prime}:=K_{0} \times \prod_{\substack{\sigma \in \Sigma \\ \sigma \neq 1}} \operatorname{SO}(p+q) .
$$

Then $K^{\prime}$ is a maximal compact subgroup of $G^{\prime}(\mathbb{R})^{0}$. Put $\tilde{X}^{\prime}:=G^{\prime}(\mathbb{R})^{0} / K^{\prime}$ and $X^{\prime}:=\Gamma^{\prime} \backslash \tilde{X}^{\prime}$. Let $\left(\rho(m), V_{\rho(m)}\right)$ be the sequence of $\mathbb{Q}$-rational representations of $G^{\prime}$ of Proposition 3.7 . Since each $\rho(m)$ is defined over $\mathbb{Q}$, there exists a free $\mathbb{Z}$-module $M_{\rho(m)}$ in $V_{\rho(m)}$ which is stable under $\rho(m)\left(\Gamma^{\prime}\right)$ and such that $M_{\rho(m)} \otimes_{\mathbb{Z}} \mathbb{Q} \cong V_{\rho(m)}$, see for example [PR, page 173]. Let $V_{\rho(m)}^{\mathbb{C}}:=V_{\rho(m)} \otimes_{\mathbb{Q}} \mathbb{C}$. Then the restriction of $\rho(m)$ to $\Gamma^{\prime}$ induces the flat complex vector bundle

$$
E_{\rho(m)}:=\tilde{X}^{\prime} \times_{\left.\rho(m)\right|_{\Gamma^{\prime}}} V_{\rho(m)}^{\mathbb{C}}
$$

over $X^{\prime}$. The decomoposition (3.24) of $\rho(m)$ induces a corresponding decomposition of $E_{\rho(m)}$ into the direct sum of complex vector bundles associated to the restriction to $\Gamma^{\prime}$ of irreducible finite-dimensional representations $\tau$ of $G^{\prime}(\mathbb{R})^{0}$. By Proposition 3.7, each $\tau$ with $[\rho(m): \tau] \neq 0$ satisfies $\tau \neq \tau_{\theta}$ and thus by [BW, Chapter VII, Theorem 6.7] one has

$$
H^{*}\left(X^{\prime} ; E_{\rho(m)}\right)=0,
$$

where $H^{*}\left(X^{\prime} ; E_{\rho(m)}\right)$ denotes the de Rham cohomology with coefficients in $E_{\rho(m)}$. Chose a Hermitian fibre metric in $E_{\rho(m)}$. Let $T_{X^{\prime}}(\rho(m))$ be the analytic torsion of $X^{\prime}$ and $\rho(m)$ 
(see (1.9)). It follows from (3.27) that $T_{X^{\prime}}(\rho(m))$ is metric independent $\mathbb{M u ̈ 1}$, Corollary 2.7]. Moreover $H^{*}\left(\Gamma^{\prime}, M_{\rho(m)}\right)$ is a finite abelian group and by (1.10) we have

$$
\log T_{X^{\prime}}(\rho(m))=\sum_{q=0}^{n}(-1)^{q+1} \log \left|H^{q}\left(\Gamma^{\prime}, M_{\rho(m)}\right)\right| .
$$

This equality reduces the proof of Theorem 1.1 to the study of the asymptotic behavior of $T_{X^{\prime}}(\rho(m))$ as $m \rightarrow \infty$, which is exactly the problem that has been dealt with in [MP]. Since $\{\rho(m)\}$ is not a sequence of representations that has been considered in [MP], we cannot apply the results of $\mathbb{M P}$ directly. We first need to reduce it to a case to which MP can be applied.

Let $\rho_{0}(m)$ be defined by (3.26) and let $T_{X^{\prime}}\left(\rho_{0}(m)\right)$ be the corresponding analytic torsion. Since $\rho(m)$ is the direct sum of $d$ copies of $\rho_{0}(m)$, we get

$$
\log T_{X^{\prime}}(\rho(m))=d \log T_{X^{\prime}}\left(\rho_{0}(m)\right)
$$

Now let $T_{X^{\prime}}^{(2)}\left(\rho_{0}(m)\right)$ be the $L^{2}$-torsion with respect to $\rho_{0}(m)$ (see $\mathbb{M P}$, section 5$]$ ). If we apply [MP, Proposition 1.2] to the irreducible components of $\rho_{0}(m)$, it follows that there exists $c>0$ such that

$$
\log T_{X^{\prime}}\left(\rho_{0}(m)\right)=\log T_{X^{\prime}}^{(2)}\left(\rho_{0}(m)\right)+O\left(e^{-c m}\right)
$$

as $m \rightarrow \infty$. Using the definition of $\rho_{0}(m)$ by (3.26), $\mathbb{M P}$, (5.21)] and $\mathbb{M P}$, Proposition 5.3], it follows that

$$
\log T_{X^{\prime}}^{(2)}\left(\rho_{0}(m)\right)=\left(\log T_{X^{\prime}}^{(2)}(\tau(m))+\log T_{X^{\prime}}^{(2)} \tau(m)_{\theta}\right)(2 \operatorname{dim} \tau(m))^{d-1} .
$$

If $C_{p, q}$ is as in (‥3), then by $\mathbb{M P}$, Proposition 6.7] one has

$$
\log T_{X^{\prime}}^{(2)}(\tau(m))=\log T_{X^{\prime}}^{(2)}\left(\tau(m)_{\theta}\right)=C_{p, q} \operatorname{vol}\left(X^{\prime}\right) m \operatorname{dim} \tau(m)+O(\operatorname{dim}(\tau(m)),
$$

as $m \rightarrow \infty$. Thus putting everything together we obtain

$$
\log T_{X^{\prime}}(\rho(m))=C_{p, q} \operatorname{vol}\left(X^{\prime}\right) m \operatorname{dim}(\rho(m))+O(\operatorname{dim}(\rho(m))),
$$

as $m \rightarrow \infty$. Since $X \cong X^{\prime}$ and $H^{*}\left(\Gamma, M_{\rho(m)}\right) \cong H^{*}\left(\Gamma^{\prime}, M_{\rho(m)}\right)$, Theorem 1.1 follows from (3.28) and the second statement of Proposition 3.7.

\section{Arithmetic subgroups OF $\mathrm{SL}_{3}(\mathbb{R})$}

Let $D$ be a nine-dimensional division algebra over $\mathbb{Q}$. Then by the Brauer-Hasse-Noether theorem [Ro], $D$ is a cyclic algebra for a cubic extension $L$ of $\mathbb{Q}$. Moreover, $L$ splits $D$, i.e. there exists an isomorphism of $L$-algebras

$$
\phi: D \otimes_{\mathbb{Q}} L \cong \operatorname{Mat}_{3 \times 3}(L) .
$$

Thus for $x \in D$ the reduced norm $N(x)$ is given by $N(x):=\operatorname{det}(\phi(x \otimes 1))$. Now let $G:=\mathrm{SL}_{1}(D)$, where

$$
\mathrm{SL}_{1}(D):=\{x \in D: N(x)=1\}
$$


Then by $[\mathbb{P R}$, 2.3.1], $G$ has a canonical structure of an algebraic group defined over $\mathbb{Q}$. We regard $\mathrm{SL}_{3}$ as an algebraic group over $\mathbb{Q}$. The isomorphism $\phi$ from (4.29) induces an isomorphism

$$
\phi: G(L) \cong \mathrm{SL}_{3}(L),
$$

i.e. $G$ is a form of $\mathrm{SL}_{3}$ over $L$. Moreover, the following Lemma holds.

Lemma 4.1. Let $\rho$ be a $\mathbb{Q}$-rational representation of $\mathrm{SL}_{3}$. Then there exists a $\mathbb{Q}$-rational representation of $G$ which over $L$ is equivalent to $\rho \circ \phi$.

Proof. By [PR, Proposition 2.17], $G$ is an inner form of $\mathrm{SL}_{3}$. Thus the proof of Lemma 3.1 in MaMü can be generalized without difficulties to prove the Lemma.

Let $\mathfrak{o}$ be an order in $D$, i.e., $\mathfrak{o}$ is a free $\mathbb{Z}$-submodule of $D$ which is generated by a $\mathbb{Z}$-base of $D$ and which is also a subring of $D$. Put

$$
\mathfrak{o}^{1}:=\{x \in \mathfrak{o}: N(x)=1\} .
$$

The left regular representation of $D$ on itself induces a $\mathbb{Q}$-rational representation of $G$ on $D$, see $[\mathbb{P R}, 2.3 .1]$ and the stabilizer of $\mathfrak{o}$ is $\mathfrak{o}^{1}$. Thus $\mathfrak{o}^{1}$ is arithmetic subgroup of $G(\mathbb{Q})$. Put

$$
\Gamma:=\phi\left(\mathfrak{o}^{1}\right)
$$

Then $\Gamma$ is an arithmetic subgroup of $\mathrm{SL}_{3}(\mathbb{R})$. Moreover, the following lemma holds.

Lemma 4.2. The group $\Gamma$ is a cocompact subgroup of $\mathrm{SL}_{3}(\mathbb{R})$.

Proof. By $[\mathrm{PR}$, Proposition 2.12], $G$ is anisotropic over $\mathbb{Q}$. Thus the proposition follows from [BoHa, Lemma 11.4, Theorem 11.8].

Let $T$ be the standard maximal torus in $\mathrm{SL}_{3}$ consisting of the diagonal matrices of determinant 1. Then $T$ is defined over $\mathbb{Q}$ and is $\mathbb{Q}$-split. Let $\mathfrak{t}$ be the Lie-algebra of $T(\mathbb{R})$ consisting of all diagonal matrices of trace 0 . Let $e_{i} \in \mathfrak{t}^{*}$ be defined by $e_{i}\left(\operatorname{diag}\left(t_{1}, t_{2}, t_{3}\right)\right):=$ $\sum_{j=1}^{3} \delta_{i, j} t_{j}$. Then with respect to the standard odering of the roots of $\mathfrak{t}_{\mathbb{C}}$ in $\mathfrak{s l}_{3, \mathbb{C}}$ the fundamental weights $\omega_{1}, \omega_{2} \in \tilde{\mathfrak{t}}_{\mathbb{C}}^{*}$ are given by

$$
\omega_{1}=\frac{2}{3}\left(e_{1}-e_{2}\right)+\frac{1}{3}\left(e_{2}-e_{3}\right) ; \quad \omega_{2}=\frac{1}{3}\left(e_{1}-e_{2}\right)+\frac{2}{3}\left(e_{2}-e_{3}\right) .
$$

The finite-dimensional irreducible representations $\tau$ of $\mathrm{SL}_{3}(\mathbb{R})$ are parametrized by their highest weights $\Lambda_{\tau}=m_{1} \omega_{1}+m_{2} \omega_{2}$. If $\theta$ is the standard Cartan involution of $\mathrm{SL}_{3}(\mathbb{R})$, then the highest weight of the representation $\tau_{\theta}:=\tau \circ \theta$ is given by $\Lambda_{\tau_{\theta}}=m_{2} \omega_{1}+m_{1} \omega_{2}$.

Proposition 4.3. Let $\tau$ be a finite-dimensional irreducible representation of $\mathrm{SL}_{3}(\mathbb{R})$ on a finite-dimensional vector space $V_{\tau}$. Then there exists a lattice $M$ in $V_{\tau}$ which is invariant under $\tau(\Gamma)$. 
Proof. Since $T$ is $\mathbb{Q}$-split $\tau$ is defined over $\mathbb{Q}[\Gamma i][$ Proposition 2.3]. By Lemma 4.1, there exists a rational representation $\tau^{\prime}$ of $G$ on a finite-dimensional $\mathbb{Q}$-vector space $V\left(\tau^{\prime}\right)$ which over $L$ is equivalent to $\tau \circ \phi$. Since $\mathfrak{o}^{1}$ is an arithmetic subgroup of $G(\mathbb{Q})$, there exists a lattice in $V\left(\tau^{\prime}\right)$ which is stable under $\tau^{\prime}\left(\mathfrak{o}^{1}\right)$, see for example $[\mathbb{P R}$, page 173]. Since $\Gamma=\phi\left(\mathfrak{o}^{1}\right)$, the Proposition follows.

We can now turn to the proof of Theorem 1.3. Let $\tilde{X}=\mathrm{SL}_{3}(\mathbb{R}) / \mathrm{SO}(3)$ and $X=\Gamma \backslash \tilde{X}$, where $\Gamma \subset \mathrm{SL}_{3}(\mathbb{R})$ is an arithmetic subgroup as above. Chose a $\mathrm{SL}_{3}(\mathbb{R})$-invariant metric on $\widetilde{X}$ and equip $X$ with the induced metric. Let $\Lambda \in \mathfrak{h}_{\mathbb{C}}^{*}$ be a highest weight. Assume that $\Lambda$ satisfies $\Lambda \neq \Lambda_{\theta}$. Then the same holds for each weight $m \Lambda, m \in \mathbb{N}$. Let $\tau_{\Lambda}(m)$ be the irreducible finite-dimensional representation on $V_{\Lambda}(m)$ with highest weight $m \Lambda$. Let $E_{\tau_{\Lambda}(m)}$ be the flat vector bundle over $X$ associated to $\tau_{\Lambda}(m)$. By [BW], Chapter VII, Theorem 6.7] we have

$$
H^{*}\left(X, E_{\tau_{\Lambda}(m)}\right)=0 .
$$

Let $T_{X}\left(\tau_{\lambda}(m)\right)$ be the analytic torsion with respect to any Hermitian fibre metric in $E_{\tau_{\Lambda}(m)}$. By (4.31) and [Mü1, Corollary 2.7], $T_{X}\left(\tau_{\Lambda}(m)\right)$ is independent of the choice of metrics on $X$ and in $E_{\tau_{\Lambda}(m)}$. Let $M_{\Lambda}(m) \subset V_{\Lambda}(m)$ be an arithmetic $\Gamma$-module, which exists by Proposition 4.3. By (4.31), $H^{*}\left(X, M_{\Lambda}(m)\right)$ is a finite abelian group and by (1.10) we have

$$
\log T_{X}\left(\tau_{\lambda}(m)\right)=\sum_{q=0}^{5}(-1)^{q+1} \log \left|H^{q}\left(X, M_{\Lambda}(m)\right)\right| .
$$

Using Theorem 1.1 and Corollary 1.5 of $[\mathrm{MP}$, the proof of Theorem 1.3 follows.

\section{REFERENCES}

[Bo1] A. Borel, Compact Clifford-Klein forms of symmetric spaces, Topology 2, 1963, 111-122

[Bo2] A. Borel, Linear Algebraic groups. Second edition Graduate Texts in Mathematics, 126. SpringerVerlag, New York, 1991

[BoHa] A. Borel, Harish-Chandra, Arithmetic subgroups of algebraic groups, Ann. of Math. (2) 75, 1962, 485-535

[Br] K.S. Brown, Cohomology of groups. Corrected reprint of the 1982 original, Graduate Texts in Mathematics, 87. Springer-Verlag, New York, 1994

[BV] N. Bergeron, A. Venkatesh, The asymptotic growth of torsion homology for arithmetic groups, http://arxiv.org/abs/1004.1083 (2010).

[BW] A. Borel, N. Wallach Continuous cohomology, discrete subgroups, and representations of reductive groups, Princeton University Press, Princeton, 1980.

[GW] R. Goodman, N. Wallach Representations and invariants of the classical groups, Encyclopedia of Mathematics and its Applications, 68. Cambridge University Press, Cambridge, 1998.

[MaMü] S. Marshall, W. Müller, On the torsion in the cohomology of arithmetic hyperbolic 3-manifolds, Preprint 2011, arXiv:1103.2262.

[Mill] J. Millson, On the first Betti number of a constant negatively curved manifold, Ann. of Math. (2) 104 (1976)

[MP] W. Müller, J. Pfaff, Analytic torsion and $L^{2}$-torsion of compact locally symmetric manifolds, Preprint 2012, arXiv:1204.0659., to appear in J. Diff. Geom.

[Mü1] W. Müller, Analytic torsion and R-torsion for unimodular representations, J. Amer. Math. Soc. 6 (1993), 721-753. 
[PR] V. P. Platonov, S.A. Rapinchuk, Algebraic groups and number theory. Translated from the 1991 Russian original by Rachel Rowen, Pure and Applied Mathematics, 139. Academic Press, Inc., Boston, MA, 1994

[Ro] P. Roquette, The Brauer-Hasse-Noether theorem in historical perspective, Schriften der Mathematisch-Naturwissenschaftlichen Klasse der Heidelberger Akademie der Wissenschaften, 15. Springer-Verlag, Berlin, 2005

[Sch] J.Schwermer, Geometric cycles, arithmetic groups and their cohomology, Bull. Amer. Math. Soc. (N.S.) 47 (2010), no. 2, 187-279

[Ti] J.Tits, Représentations linéaires irréductibles d'un groupe réductif sur un corps quelconque, J. Reine Angew. Math. 2471971 196-220

[Tu] V. G. Turaev, Reidemeister torsion in knot theory, Russian Math. Surveys 41:1 (1986), 119-182.

[Wei] A. Weil, Adeles and algebraic groups. With appendices by M. Demazure and Takashi Ono, Progress in Mathematics, 23. Birkhäuser, Boston, Mass., 1982

[Zi] R. J. Zimmer, Ergodic theory and semisimple groups, Monographs in Mathematics, 81. Birkhäuser Verlag, Basel, 1984

Universität Bonn, Mathematisches Institut, Endenicher Allee 60, D - 53115 Bonn, GerMANY

E-mail address: mueller@math.uni-bonn.de

Universität Bonn, Mathematisches Institut, Q Endenicher Alle 60, D - 53115 Bonn, Germany

E-mail address: pfaff@math.uni-bonn.de 